\title{
The additivity of ring distortions in halogen-substituted aromatics: a gas-phase electron diffraction and computational study
}

Derek A. Wann, Sarah L. Masters (née Hinchley), Heather E. Robertson and David W. H.

Rankin

\section{Supporting Information}

Table S1 Nozzle-to-plate distances / mm, nozzle and sample temperatures / K, weighting functions $/ \mathrm{nm}^{-1}$, scale factors, correlation parameters and electron wavelengths, $\lambda / \mathrm{pm}$, used in the electron diffraction studies of 1,2,3-trifluorobenzene, 1, 1,3,5trifluorobenzene, 2, 2,6-difluoropyridine, 3, and 2,6-dichloropyridine, 4.

\begin{tabular}{llllllllllll}
\hline & $\begin{array}{l}\text { Nozzle-to-plate } \\
\text { distance }^{a}\end{array}$ & $T_{\text {nozzle }}$ & $T_{\text {sample }}$ & $\Delta s$ & $s_{\min }$ & $s w_{1}$ & $s w_{2}$ & $s_{\max }$ & $\begin{array}{l}\text { Scale } \\
\text { factor }^{b}\end{array}$ & $\begin{array}{l}\text { Correlation } \\
\text { parameter }\end{array}$ & $\lambda$ \\
\hline $\mathbf{1}$ & 128.18 & 297 & 297 & 4 & 80 & 100 & 256 & 300 & $0.704(8)$ & 0.458 & 6.016 \\
& 285.34 & 297 & 297 & 2 & 20 & 40 & 112 & 130 & $0.749(2)$ & 0.485 & 6.016 \\
$\mathbf{2}$ & 128.16 & 297 & 297 & 4 & 80 & 100 & 276 & 320 & $0.578(10)$ & 0.426 & 6.016 \\
& 285.49 & 297 & 297 & 2 & 20 & 40 & 118 & 138 & $0.661(5)$ & 0.491 & 6.016 \\
$\mathbf{3}$ & 86.34 & 373 & 356 & 4 & 120 & 140 & 276 & 320 & $0.695(17)$ & 0.449 & 6.016 \\
& 256.41 & 336 & 325 & 2 & 20 & 40 & 128 & 148 & $0.666(4)$ & 0.471 & 6.016 \\
$\mathbf{4}$ & 98.15 & 406 & 385 & 2 & 100 & 120 & 276 & 320 & $0.864(20)$ & 0.348 & 6.130 \\
& 261.96 & 384 & 373 & 1 & 20 & 40 & 120 & 140 & $0.860(5)$ & 0.489 & 6.130 \\
\hline
\end{tabular}

${ }^{a}$ Determined by reference to the scattering pattern of benzene. ${ }^{b}$ Values in parentheses are the estimated standard deviations. 
Table S2 Interatomic distances $\left(r_{\mathrm{a}} / \mathrm{pm}\right)$, perpendicular correction values $\left(k_{\mathrm{h} 1} / \mathrm{pm}\right)$ and calculated and refined amplitudes of vibration $\left(u_{\mathrm{h} 1} / \mathrm{pm}\right)$ for the restrained GED structure of 1,2,3-trifluorobenzene, $\mathbf{1}^{a}$

\begin{tabular}{|c|c|c|c|c|c|c|}
\hline & Atom pair & $r_{\mathrm{a}}$ & $u_{\mathrm{h} 1}(\exp .)^{b}$ & Restraint & $k_{\mathrm{h} 1}$ & $u_{\mathrm{h} 1}(\text { calc. })^{b}$ \\
\hline$u_{1}$ & $\mathrm{C}(4)-\mathrm{H}(4)$ & $107.8(6)$ & $8.5(6)$ & $7.3(7)$ & 0.3 & 7.3 \\
\hline$u_{2}$ & $\mathrm{C}(5)-\mathrm{H}(5)$ & $107.8(6)$ & $8.5\left(\right.$ tied to $\left.u_{1}\right)$ & - & 0.3 & 7.3 \\
\hline$u_{3}$ & $\mathrm{C}(2)-\mathrm{F}(2)$ & $132.4(3)$ & $3.5\left(\right.$ tied to $u_{4}$ ) & -- & 0.1 & 4.1 \\
\hline$u_{4}$ & $\mathrm{C}(1)-\mathrm{F}(1)$ & $133.2(3)$ & $3.6(3)$ & $4.1(4)$ & 0.1 & 4.1 \\
\hline$u_{5}$ & $C(3)-C(4)$ & $139.2(2)$ & 3.8(tied to $\left.u_{4}\right)$ & - & 0.1 & 4.4 \\
\hline$u_{6}$ & $\mathrm{C}(1)-\mathrm{C}(2)$ & $139.5(2)$ & 3.8(tied to $\left.u_{4}\right)$ & - & 0.0 & 4.4 \\
\hline$u_{7}$ & $C(4)-C(5)$ & $140.3(2)$ & $3.9\left(\right.$ tied to $\left.u_{4}\right)$ & - & 0.2 & 4.4 \\
\hline$u_{8}$ & $\mathrm{C}(1) \cdots \mathrm{H}(6)$ & $211.7(10)$ & 9.5 (fixed) & - & -0.3 & 9.5 \\
\hline$u_{9}$ & $\mathrm{C}(4) \cdots \mathrm{H}(5)$ & $215.4(8)$ & 9.4(fixed) & - & -0.2 & 9.4 \\
\hline$u_{10}$ & $\mathrm{C}(5) \cdots \mathrm{H}(4)$ & 217.2(5) & 9.4(fixed) & - & -0.2 & 9.4 \\
\hline$u_{11}$ & $C(2) \cdots F(1)$ & $234.0(3)$ & $5.8(2)$ & - & -0.3 & 5.5 \\
\hline$u_{12}$ & $C(4) \cdots F(3)$ & $237.0(3)$ & 5.8(tied to $\left.u_{11}\right)$ & - & -0.2 & 5.5 \\
\hline$u_{13}$ & $\mathrm{C}(1) \cdots \mathrm{F}(2)$ & $235.4(5)$ & 5.8(tied to $\left.u_{11}\right)$ & - & -0.3 & 5.6 \\
\hline$u_{14}$ & $C(1) \cdots C(3)$ & $240.8(10)$ & 5.3(tied to $\left.u_{11}\right)$ & - & -0.3 & 5.1 \\
\hline$u_{15}$ & $C(5) \cdots C(1)$ & $242.0(10)$ & 5.4(tied to $\left.u_{11}\right)$ & - & -0.1 & 5.2 \\
\hline$u_{16}$ & $C(4) \cdots C(2)$ & $241.6(6)$ & 5.4(tied to $\left.u_{11}\right)$ & - & -0.2 & 5.2 \\
\hline$u_{17}$ & $C(4) \cdots C(6)$ & $241.9(16)$ & $5.5\left(\right.$ tied to $\left.u_{11}\right)$ & - & 0.0 & 5.2 \\
\hline$u_{18}$ & $\mathrm{H}(4) \cdots \mathrm{H}(5)$ & 251.1(12) & 15.2(fixed) & - & -0.1 & 15.2 \\
\hline$u_{19}$ & $H(4) \cdots F(3)$ & $258.4(14)$ & 12.8(fixed) & - & -0.2 & 12.8 \\
\hline$u_{20}$ & $F(1) \cdots F(2)$ & 269.1(4) & $8.7(4)$ & - & -0.4 & 9.2 \\
\hline$u_{21}$ & $C(4) \cdots C(1)$ & $278.5(11)$ & $5.5\left(\right.$ tied to $\left.u_{20}\right)$ & - & -0.2 & 5.8 \\
\hline$u_{22}$ & $C(5) \cdots C(2)$ & 279.9(19) & 5.5(tied to $\left.u_{20}\right)$ & - & -0.3 & 5.8 \\
\hline$u_{23}$ & $\mathrm{C}(1) \cdots \mathrm{H}(5)$ & $339.2(12)$ & 9.0 (fixed) & - & -0.8 & 9.0 \\
\hline$u_{24}$ & $\mathrm{C}(2) \cdots \mathrm{H}(4)$ & $337.1(8)$ & 9.1 (fixed) & - & -1.0 & 9.1 \\
\hline$u_{25}$ & $\mathrm{C}(4) \cdots \mathrm{H}(6)$ & $340.5(12)$ & 9.0 (fixed) & - & -0.8 & 9.0 \\
\hline$u_{26}$ & $C(1) \cdots F(3)$ & $360.9(8)$ & $6.8(2)$ & - & -0.7 & 5.6 \\
\hline$u_{27}$ & $C(5) \cdots F(1)$ & $364.0(7)$ & 6.8(tied to $\left.u_{26}\right)$ & - & -0.5 & 5.6 \\
\hline$u_{28}$ & $C(4) \cdots F(2)$ & $361.9(6)$ & $6.8\left(\right.$ tied to $\left.u_{26}\right)$ & - & -0.6 & 5.6 \\
\hline$u_{29}$ & $\mathrm{C}(1) \cdots \mathrm{H}(4)$ & $385.6(13)$ & 8.9 (fixed) & - & -1.1 & 8.9 \\
\hline$u_{30}$ & $\mathrm{C}(2) \cdots \mathrm{H}(5)$ & $387.1(20)$ & 8.9 (fixed) & - & -1.2 & 8.9 \\
\hline$u_{31}$ & $\mathrm{C}(4) \cdots \mathrm{F}(1)$ & $411.2(10)$ & $7.4(3)$ & - & -0.7 & 5.9 \\
\hline$u_{32}$ & $C(5) \cdots F(2)$ & $411.8(19)$ & 7.4(tied to $\left.u_{31}\right)$ & - & -0.8 & 5.9 \\
\hline$u_{33}$ & $\mathrm{H}(4) \cdots \mathrm{H}(6)$ & $431.7(12)$ & 12.3(fixed) & - & -1.6 & 12.3 \\
\hline$u_{34}$ & $\mathrm{H}(4) \cdots \mathrm{F}(2)$ & $446.4(8)$ & 10.1 (fixed) & - & -1.4 & 10.1 \\
\hline$u_{35}$ & $\mathrm{H}(5) \cdots \mathrm{F}(1)$ & $451.4(10)$ & 10.0 (fixed) & - & -1.2 & 10.0 \\
\hline$u_{36}$ & $F(1) \cdots F(3)$ & $467.5(7)$ & $7.9(7)$ & - & -1.2 & 6.7 \\
\hline$u_{37}$ & $H(4) \cdots F(1)$ & $518.2(12)$ & 9.0 (fixed) & - & -1.7 & 9.0 \\
\hline$u_{38}$ & $\mathrm{H}(5) \cdots \mathrm{F}(2)$ & $518.9(20)$ & 9.0 (fixed) & - & -1.8 & 9.0 \\
\hline
\end{tabular}

${ }^{a}$ Estimated standard deviations, as obtained in the least-squares refinement, are given in parentheses. ${ }^{b}$ Amplitudes not refined were fixed at the values obtained using the force field calculated at RHF/6-31G*. 
Table S3 Interatomic distances $\left(r_{\mathrm{a}} / \mathrm{pm}\right)$, perpendicular correction values $\left(k_{\mathrm{h} 1} / \mathrm{pm}\right)$ and calculated and refined amplitudes of vibration $\left(u_{\mathrm{h} 1} / \mathrm{pm}\right)$ for the restrained GED structure of 1,3,5-trifluorobenzene, $\mathbf{2}^{a}{ }^{a}$

\begin{tabular}{lllllcc}
\hline & Atom pair & $r_{\mathrm{a}}$ & $u_{\mathrm{h} 1}(\text { exp. })^{b}$ & Restraint & $k_{\mathrm{h} 1}$ & $u_{\mathrm{h} 1}(\text { calc. })^{b}$ \\
\hline$u_{1}$ & $\mathrm{C}(2)-\mathrm{H}(2)$ & $106.5(10)$ & $7.8(6)$ & $7.3(7)$ & 0.3 & 7.3 \\
$u_{2}$ & $\mathrm{C}(1)-\mathrm{F}(1)$ & $133.2(2)$ & 3.3 (tied to u3) & - & 0.1 & 4.1 \\
$u_{3}$ & $\mathrm{C}(1)-\mathrm{C}(2)$ & $139.3(1)$ & $3.5(3)$ & - & 0.1 & 4.4 \\
$u_{4}$ & $\mathrm{C}(1) \cdots \mathrm{H}(2)$ & $215.4(8)$ & $8.0(15)$ & - & -0.4 & 9.5 \\
$u_{5}$ & $\mathrm{C}(2) \cdots \mathrm{F}(1)$ & $234.1(1)$ & $5.4(2)$ & - & -0.2 & 5.5 \\
$u_{6}$ & $\mathrm{C}(1) \cdots \mathrm{C}(3)$ & $237.3(3)$ & 5.0 (tied to u5) & - & -0.2 & 5.1 \\
$u_{7}$ & $\mathrm{C}(2) \cdots \mathrm{C}(4)$ & $245.2(3)$ & 5.1 (tied to u5) & - & -0.1 & 5.2 \\
$u_{8}$ & $\mathrm{~F}(1) \cdots \mathrm{H}(2)$ & $259.8(4)$ & $10.2(14)$ & - & -0.2 & 12.8 \\
$u_{9}$ & $\mathrm{C}(1) \cdots \mathrm{C}(4)$ & $278.6(3)$ & $5.6(5)$ & - & -0.2 & 5.8 \\
$u_{10}$ & $\mathrm{C}(2) \cdots \mathrm{H}(4)$ & $342.0(10)$ & 9.1 (fixed) & - & -0.9 & 9.1 \\
$u_{11}$ & $\mathrm{C}(1) \cdots \mathrm{F}(3)$ & $359.0(1)$ & $6.7(2)$ & - & -0.6 & 5.6 \\
$u_{12}$ & $\mathrm{C}(1) \cdots \mathrm{H}(4)$ & $385.6(10)$ & 8.9 (fixed) & - & -1.2 & 8.9 \\
$u_{13}$ & $\mathrm{C}(2) \cdots \mathrm{F}(5)$ & $411.9(2)$ & $7.4(4)$ & - & -0.7 & 5.8 \\
$u_{14}$ & $\mathrm{H}(2) \cdots \mathrm{H}(4)$ & $430.4(17)$ & 12.5 (fixed) & - & -1.8 & 12.5 \\
$u_{15}$ & $\mathrm{~F}(1) \cdots \mathrm{F}(3)$ & $468.2(2)$ & $8.2(3)$ & - & -1.0 & 6.5 \\
$u_{16}$ & $\mathrm{~F}(1) \cdots \mathrm{H}(4)$ & $518.9(10)$ & $9.0($ fixed) & - & -1.8 & 9.0 \\
\hline
\end{tabular}

${ }^{a}$ Estimated standard deviations, as obtained in the least-squares refinement, are given in parentheses. ${ }^{b}$ Amplitudes not refined were fixed at the values obtained using the force field calculated at RHF/6-31G*. 
Table S4 Interatomic distances $\left(r_{\mathrm{a}} / \mathrm{pm}\right)$, perpendicular correction values $\left(k_{\mathrm{h} 1} / \mathrm{pm}\right)$ and calculated and refined amplitudes of vibration $\left(u_{\mathrm{h} 1} / \mathrm{pm}\right)$ for the restrained GED structure of 2,6-difluoropyridine, $\mathbf{3}^{a}$

\begin{tabular}{|c|c|c|c|c|c|c|}
\hline & Atom pair & $r_{\mathrm{a}}$ & $u_{\mathrm{h} 1}(\exp .)^{b}$ & Restraint & $k_{\mathrm{h} 1}$ & $u_{\mathrm{h} 1}(\text { calc. })^{b}$ \\
\hline$u_{1}$ & $\mathrm{C}(3)-\mathrm{H}(3)$ & $108.2(4)$ & $7.3(6)$ & $7.3(7)$ & 0.3 & 7.3 \\
\hline$u_{2}$ & $\mathrm{C}(4)-\mathrm{H}(4)$ & $108.2(4)$ & 7.4(tied to $\left.u_{1}\right)$ & - & 0.4 & 7.3 \\
\hline$u_{3}$ & $\mathrm{C}(2)-\mathrm{N}(1)$ & $131.8(4)$ & 4.0(tied to $\left.u_{4}\right)$ & - & -0.1 & 4.2 \\
\hline$u_{4}$ & $\mathrm{C}(2)-\mathrm{F}(2)$ & $133.6(3)$ & $4.0(2)$ & - & 0.1 & 4.1 \\
\hline$u_{5}$ & $C(2)-C(3)$ & 139.2(2) & 4.3(tied to $\left.u_{4}\right)$ & - & 0.1 & 4.4 \\
\hline$u_{6}$ & $\mathrm{C}(3)-\mathrm{C}(4)$ & $139.9(2)$ & 4.3(tied to $\left.u_{4}\right)$ & - & 0.2 & 4.4 \\
\hline$u_{7}$ & $\mathrm{C}(3) \cdots \mathrm{H}(4)$ & $215.0(4)$ & 9.5 (fixed) & - & -0.1 & 9.5 \\
\hline$u_{8}$ & $\mathrm{C}(6) \cdots \mathrm{H}(5)$ & 215.2(7) & 9.5(fixed) & - & -0.3 & 9.5 \\
\hline$u_{9}$ & $\mathrm{C}(4) \cdots \mathrm{H}(3)$ & $217.9(6)$ & 9.4(fixed) & - & -0.2 & 9.4 \\
\hline$u_{10}$ & $C(6) \cdots C(2)$ & $222.8(6)$ & $5.5\left(\right.$ tied to $\left.u_{11}\right)$ & - & -0.3 & 5.0 \\
\hline$u_{11}$ & $\mathrm{~N}(1) \cdots \mathrm{F}(6)$ & $224.3(2)$ & $5.9(3)$ & - & -0.2 & 5.3 \\
\hline$u_{12}$ & $C(3) \cdots F(2)$ & $234.1(5)$ & $5.9(3)$ & - & -0.2 & 5.6 \\
\hline$u_{13}$ & $C(4) \cdots C(6)$ & $236.6(4)$ & $5.5\left(\right.$ tied to $\left.u_{12}\right)$ & - & -0.2 & 5.2 \\
\hline$u_{14}$ & $\mathrm{C}(3) \cdots \mathrm{N}(1)$ & $241.5(5)$ & 5.4(tied to $\left.u_{12}\right)$ & - & -0.2 & 5.1 \\
\hline$u_{15}$ & $C(3) \cdots C(5)$ & $242.0(7)$ & 5.6(tied to $\left.u_{12}\right)$ & - & 0.0 & 5.3 \\
\hline$u_{16}$ & $\mathrm{H}(3) \cdots \mathrm{H}(4)$ & 252.0(9) & 15.3(fixed) & - & -0.1 & 15.3 \\
\hline$u_{17}$ & $\mathrm{H}(3) \cdots \mathrm{F}(2)$ & $258.7(12)$ & 12.9(fixed) & - & -0.2 & 12.9 \\
\hline$u_{18}$ & $C(3) \cdots C(6)$ & $270.6(4)$ & $7.4(6)$ & - & -0.3 & 5.7 \\
\hline$u_{19}$ & $\mathrm{C}(4) \cdots \mathrm{N}(1)$ & $278.8(8)$ & $7.8\left(\right.$ tied to $\left.u_{18}\right)$ & - & -0.4 & 6.0 \\
\hline$u_{20}$ & $\mathrm{C}(6) \cdots \mathrm{H}(4)$ & $335.2(6)$ & 9.0 (fixed) & - & -0.9 & 9.0 \\
\hline$u_{21}$ & $\mathrm{~N}(1) \cdots \mathrm{H}(3)$ & $336.7(7)$ & 9.1(fixed) & - & -1.0 & 9.1 \\
\hline$u_{22}$ & $\mathrm{C}(3) \cdots \mathrm{H}(5)$ & $341.2(7)$ & 9.0 (fixed) & - & -0.8 & 9.0 \\
\hline$u_{23}$ & $\mathrm{C}(6) \cdots \mathrm{F}(2)$ & $342.9(3)$ & $7.3(5)$ & - & -0.7 & 5.5 \\
\hline$u_{24}$ & $C(4) \cdots F(6)$ & $358.4(4)$ & $7.7\left(\right.$ tied to $\left.u_{23}\right)$ & - & -0.6 & 5.7 \\
\hline$u_{25}$ & $\mathrm{C}(6) \cdots \mathrm{H}(3)$ & $377.8(6)$ & 8.9 (fixed) & - & -1.2 & 8.9 \\
\hline$u_{26}$ & $\mathrm{~N}(1) \cdots \mathrm{H}(4)$ & 386.3(9) & 9.1(fixed) & - & -1.2 & 9.1 \\
\hline$u_{27}$ & $\mathrm{C}(3) \cdots \mathrm{F}(6)$ & $403.8(4)$ & $7.5(4)$ & - & -0.8 & 5.9 \\
\hline$u_{28}$ & $\mathrm{H}(3) \cdots \mathrm{H}(5)$ & $433.2(12)$ & 12.2(fixed) & - & -1.6 & 12.2 \\
\hline$u_{29}$ & $F(6) \cdots F(2)$ & $447.9(4)$ & 10.8(fixed) & - & -1.1 & 6.6 \\
\hline$u_{30}$ & $\mathrm{H}(4) \cdots \mathrm{F}(6)$ & $447.3(5)$ & 10.0(fixed) & - & -1.3 & 10.0 \\
\hline$u_{31}$ & $\mathrm{H}(3) \cdots \mathrm{F}(6)$ & $510.7(5)$ & 9.0(fixed) & - & -1.9 & 9.0 \\
\hline
\end{tabular}

${ }^{a}$ Estimated standard deviations, as obtained in the least-squares refinement, are given in parentheses. ${ }^{b}$ Amplitudes not refined were fixed at the values obtained using the force field calculated at RHF/6-31G*. 
Table S5 Interatomic distances $\left(r_{\mathrm{a}} / \mathrm{pm}\right)$, perpendicular correction values $\left(k_{\mathrm{h} 1} / \mathrm{pm}\right)$ and calculated and refined amplitudes of vibration $\left(u_{\mathrm{h} 1} / \mathrm{pm}\right)$ for the restrained GED structure of 2,6-dichloropyridine, $\mathbf{4}^{a}$

\begin{tabular}{|c|c|c|c|c|c|c|}
\hline & Atom pair & $r_{\mathrm{a}}$ & $u_{\mathrm{h} 1}(\text { exp. })^{b}$ & Restraint & $k_{\mathrm{h} 1}$ & $u_{\mathrm{h} 1}$ (calc.) $^{b}$ \\
\hline$u_{1}$ & $\mathrm{C}(3)-\mathrm{H}(3)$ & $108.0(2)$ & $6.9(5)$ & $7.3(7)$ & 0.2 & 7.3 \\
\hline$u_{2}$ & $\mathrm{C}(4)-\mathrm{H}(4)$ & $107.9(2)$ & $6.9\left(\right.$ tied to $\left.u_{1}\right)$ & - & 0.1 & 7.3 \\
\hline$u_{3}$ & $\mathrm{C}(2)-\mathrm{N}(1)$ & $132.8(3)$ & $5.3(2)$ & 一 & 0.3 & 4.3 \\
\hline$u_{4}$ & $C(2)-C(3)$ & $138.6(2)$ & $5.5\left(\right.$ tied to $\left.u_{3}\right)$ & 一 & 0.4 & 4.5 \\
\hline$u_{5}$ & $\mathrm{C}(3)-\mathrm{C}(4)$ & $138.5(2)$ & $5.5\left(\right.$ tied to $\left.u_{3}\right)$ & 一 & 0.0 & 4.5 \\
\hline$u_{6}$ & $\mathrm{C}(2)-\mathrm{Cl}(2)$ & 171.7(1) & $5.8(2)$ & 一 & 0.1 & 4.7 \\
\hline$u_{7}$ & $\mathrm{C}(5) \cdots \mathrm{H}(4)$ & $213.9(3)$ & 9.5(fixed) & - & -0.1 & 9.5 \\
\hline$u_{8}$ & $\mathrm{C}(2) \cdots \mathrm{H}(3)$ & $213.5(10)$ & 9.4(fixed) & 一 & -0.1 & 9.4 \\
\hline$u_{9}$ & $\mathrm{C}(4) \cdots \mathrm{H}(5)$ & $216.7(9)$ & 9.4(fixed) & 一 & -0.7 & 9.4 \\
\hline$u_{10}$ & $C(2) \cdots C(6)$ & $225.3(2)$ & 5.4(tied to $\left.u_{12}\right)$ & 一 & -0.1 & 5.1 \\
\hline$u_{11}$ & $C(4) \cdots C(2)$ & $236.0(3)$ & 5.6(tied to $\left.u_{12}\right)$ & 一 & -0.2 & 5.3 \\
\hline$u_{12}$ & $C(5) \cdots N(1)$ & $240.1(3)$ & $5.5(2)$ & - & -0.2 & 5.2 \\
\hline$u_{13}$ & $C(3) \cdots C(5)$ & $240.3(4)$ & 5.6(tied to $\left.u_{12}\right)$ & - & 0.1 & 5.3 \\
\hline$u_{14}$ & $\mathrm{H}(5) \cdots \mathrm{H}(4)$ & $250.6(14)$ & 15.2(fixed) & - & -0.6 & 15.2 \\
\hline$u_{15}$ & $\mathrm{~N}(1) \cdots \mathrm{Cl}(2)$ & $258.6(1)$ & $6.7(2)$ & - & -0.2 & 6.0 \\
\hline$u_{16}$ & $\mathrm{C}(5) \cdots \mathrm{Cl}(6)$ & $267.5(2)$ & 7.0(tied to $\left.u_{15}\right)$ & 一 & -0.6 & 6.3 \\
\hline$u_{17}$ & $C(5) \cdots C(2)$ & $270.6(2)$ & $6.5\left(\right.$ tied to $\left.u_{15}\right)$ & 一 & -0.1 & 5.8 \\
\hline$u_{18}$ & $\mathrm{C}(4) \cdots \mathrm{N}(1)$ & $276.9(4)$ & $6.8\left(\right.$ tied to $\left.u_{15}\right)$ & - & -0.4 & 6.0 \\
\hline$u_{19}$ & $\mathrm{H}(5) \cdots \mathrm{Cl}(6)$ & $280.1(16)$ & 13.6(fixed) & - & -1.3 & 13.6 \\
\hline$u_{20}$ & $\mathrm{~N}(1) \cdots \mathrm{H}(5)$ & $335.4(7)$ & 9.1(fixed) & - & -0.6 & 9.1 \\
\hline$u_{21}$ & $\mathrm{C}(2) \cdots \mathrm{H}(4)$ & $334.0(4)$ & 9.1(fixed) & - & -1.1 & 9.1 \\
\hline$u_{22}$ & $\mathrm{C}(5) \cdots \mathrm{H}(3)$ & $339.1(6)$ & 9.1(fixed) & - & -1.1 & 9.0 \\
\hline$u_{23}$ & $\mathrm{C}(2) \cdots \mathrm{H}(5)$ & $378.3(3)$ & 9.0(fixed) & - & -0.4 & 9.0 \\
\hline$u_{24}$ & $\mathrm{C}(2) \cdots \mathrm{Cl}(6)$ & $381.1(2)$ & $7.0(3)$ & - & -0.7 & 6.1 \\
\hline$u_{25}$ & $\mathrm{~N}(1) \cdots \mathrm{H}(4)$ & $384.6(5)$ & 9.1 (fixed) & - & -1.0 & 9.1 \\
\hline$u_{26}$ & $\mathrm{C}(4) \cdots \mathrm{F}(2)$ & $396.0(2)$ & 7.2(tied to $\left.u_{24}\right)$ & - & 0.8 & 6.2 \\
\hline$u_{27}$ & $\mathrm{H}(5) \cdots \mathrm{H}(3)$ & $431.1(15)$ & 12.3(fixed) & - & -2.1 & 12.3 \\
\hline$u_{28}$ & $\mathrm{C}(5) \cdots \mathrm{Cl}(2)$ & $442.2(2)$ & $7.7(2)$ & - & -0.4 & 6.2 \\
\hline$u_{29}$ & $\mathrm{H}(4) \cdots \mathrm{Cl}(2)$ & $480.7(3)$ & 10.6(fixed) & - & -1.6 & 10.6 \\
\hline$u_{30}$ & $\mathrm{Cl}(2) \cdots \mathrm{Cl}(6)$ & $515.3(2)$ & $10.2(2)$ & - & -0.4 & 8.4 \\
\hline$u_{31}$ & $\mathrm{H}(5) \cdots \mathrm{Cl}(6)$ & $548.4(3)$ & 9.2(fixed) & - & -2.2 & 9.2 \\
\hline
\end{tabular}

${ }^{a}$ Estimated standard deviations, as obtained in the least-squares refinement, are given in parentheses. ${ }^{b}$ Amplitudes not refined were fixed at the values obtained using the force field calculated at RHF/6-31G*. 
Table S6 Least-squares correlation matrix $(\times 100)$ for 1,2,3-trifluorobenzene, $\mathbf{1}^{a}$

\begin{tabular}{lrrrrrrr}
\hline & $p_{4}$ & $p_{8}$ & $p_{9}$ & $u_{4}$ & $u_{11}$ & $u_{20}$ & $u_{26}$ \\
\hline$p_{1}$ & -92 & & & -88 & -79 & -77 & \\
$p_{4}$ & & & & 89 & 75 & 73 & \\
$p_{7}$ & & 82 & 75 & & & & 58 \\
$p_{9}$ & & & & & & & \\
$u_{4}$ & & & & & & \\
$u_{11}$ & & & & \\
${ }^{a}$ Only elements with absolute values $>50 \%$ & are shown.
\end{tabular}

Table S7 Least-squares correlation matrix $(\times 100)$ for 1,3,5-trifluorobenzene, $2{ }^{a}$

\begin{tabular}{rrrrr}
\hline & $p_{2}$ & $u_{3}$ & $u_{5}$ & $k_{2}$ \\
\hline$p_{1}$ & -82 & 72 & & \\
$p_{2}$ & & -68 & -58 & \\
$u_{3}$ & & & 57 & 51 \\
\hline
\end{tabular}

${ }^{a}$ Only elements with absolute values $>50 \%$ are shown; $k_{2}$ is a scale factor.

Table S8 Least-squares correlation matrix $(\times 100)$ for 2,6-difluoropyridine, $\mathbf{3}^{a}$

\begin{tabular}{lrrrrr}
\hline & $p_{4}$ & $p_{9}$ & $p_{10}$ & $u_{4}$ & $k_{2}$ \\
\hline$p_{1}$ & -52 & & 75 & -70 & \\
$p_{3}$ & -53 & & & & \\
$p_{4}$ & & -62 & -61 & 60 \\
$p_{10}$ & & & -62 & 56 \\
$u_{6}$ & & & \\
${ }^{a}$ Only elements with absolute values $>50 \%$ & are shown; $k_{2}$ is a scale factor.
\end{tabular}

Table S9 Least-squares correlation matrix (×100) for 2,6-dichloropyridine, $4^{a}$

\begin{tabular}{lrrrrr}
\hline & $p_{4}$ & $p_{9}$ & $p_{10}$ & \multicolumn{1}{c}{$u_{3}$} & $k_{2}$ \\
\hline$p_{1}$ & -86 & 79 & 84 & & \\
$p_{4}$ & & -88 & -90 & 63 & \\
$p_{9}$ & & & 89 & -56 & \\
$p_{10}$ & & & -53 & \\
$u_{3}$ & & & & 68 \\
$u_{6}$ & & & 82 \\
${ }^{a}$ Only elements with absolute values $>50 \%$ & are shown; $k_{2}$ is a scale factor.
\end{tabular}


Table S10 Calculated coordinates (MP2/6-311++G**) for 1,2,3-trifluorobenzene, 1.

\begin{tabular}{lrrc}
\hline Atom & \multicolumn{1}{c}{$x$} & \multicolumn{1}{c}{$y$} & $z$ \\
\hline $\mathrm{C}(1)$ & -1.2006 & 0.0154 & 0.0000 \\
$\mathrm{C}(2)$ & 0.0000 & 0.7257 & 0.0000 \\
$\mathrm{C}(3)$ & 1.2006 & 0.0154 & 0.0000 \\
$\mathrm{C}(4)$ & 1.2162 & -1.3754 & 0.0000 \\
$\mathrm{C}(5)$ & 0.0000 & -2.0664 & 0.0000 \\
$\mathrm{C}(6)$ & -1.2162 & -1.3754 & 0.0000 \\
$\mathrm{~F}(1)$ & -2.3483 & 0.7086 & 0.0000 \\
$\mathrm{~F}(2)$ & 0.0000 & 2.0600 & 0.0000 \\
$\mathrm{~F}(3)$ & 2.3483 & 0.7086 & 0.0000 \\
$\mathrm{H}(4)$ & 2.1709 & -1.8902 & 0.0000 \\
$\mathrm{H}(5)$ & 0.0000 & -3.1515 & 0.0000 \\
$\mathrm{H}(6)$ & -2.1709 & -1.8902 & 0.0000 \\
\hline
\end{tabular}

Energy $=-528.88534$ Hartrees (corrected for ZPE).

Table S11 Calculated coordinates (MP2/6-311++G**) for 1,3,5-trifluorobenzene, 2.

\begin{tabular}{lrrc}
\hline Atom & \multicolumn{1}{c}{$x$} & \multicolumn{1}{c}{$y$} & $z$ \\
\hline $\mathrm{C}(1)$ & 0.0000 & 1.3686 & 0.0000 \\
$\mathrm{C}(2)$ & 1.2243 & 0.7068 & 0.0000 \\
$\mathrm{C}(3)$ & 1.1853 & -0.6843 & 0.0000 \\
$\mathrm{C}(4)$ & 0.0000 & -1.4137 & 0.0000 \\
$\mathrm{C}(5)$ & -1.1853 & -0.6843 & 0.0000 \\
$\mathrm{C}(6)$ & -1.2243 & 0.7068 & 0.0000 \\
$\mathrm{~F}(1)$ & 0.0000 & 2.7143 & 0.0000 \\
$\mathrm{H}(2)$ & 2.1625 & 1.2485 & 0.0000 \\
$\mathrm{~F}(3)$ & 2.3507 & -1.3571 & 0.0000 \\
$\mathrm{H}(4)$ & 0.0000 & -2.4971 & 0.0000 \\
$\mathrm{~F}(5)$ & -2.3507 & -1.3571 & 0.0000 \\
$\mathrm{H}(6)$ & -2.1625 & 1.2485 & 0.0000 \\
\hline
\end{tabular}

Energy $=-528.89699$ Hartrees (corrected for ZPE). 
Table S12 Calculated coordinates (MP2/6-311++G**) for 2,6-difluoropyridine, 3.

\begin{tabular}{lrrc}
\hline Atom & \multicolumn{1}{c}{$x$} & \multicolumn{1}{c}{$y$} & $z$ \\
\hline $\mathrm{N}(1)$ & 0.0000 & 1.0184 & 0.0000 \\
$\mathrm{C}(2)$ & 1.1190 & 0.3165 & 0.0000 \\
$\mathrm{C}(3)$ & 1.2077 & -1.0727 & 0.0000 \\
$\mathrm{C}(4)$ & 0.0000 & -1.7744 & 0.0000 \\
$\mathrm{C}(5)$ & -1.2077 & -1.0727 & 0.0000 \\
$\mathrm{C}(6)$ & -1.1190 & 0.3165 & 0.0000 \\
$\mathrm{~F}(2)$ & 2.2506 & 1.0322 & 0.0000 \\
$\mathrm{H}(3)$ & 2.1732 & -1.5641 & 0.0000 \\
$\mathrm{H}(4)$ & 0.0000 & -2.8601 & 0.0000 \\
$\mathrm{H}(5)$ & -2.1732 & -1.5641 & 0.0000 \\
$\mathrm{~F}(6)$ & -2.2506 & 1.0322 & 0.0000 \\
\hline
\end{tabular}

Energy $=-445.85795$ Hartrees (corrected for ZPE).

Table S13 Calculated coordinates (MP2/6-311++G**) for 2,6-dichloropyridine, 4.

\begin{tabular}{lrrc}
\hline Atom & \multicolumn{1}{c}{$x$} & \multicolumn{1}{c}{$y$} & \multicolumn{1}{c}{$z$} \\
\hline $\mathrm{N}(1)$ & 0.0000 & 0.6999 & 0.0000 \\
$\mathrm{C}(2)$ & -1.1341 & 0.0017 & 0.0000 \\
$\mathrm{C}(3)$ & -1.2059 & -1.3942 & 0.0000 \\
$\mathrm{C}(4)$ & 0.0000 & -2.0971 & 0.0000 \\
$\mathrm{C}(5)$ & 1.2059 & -1.3942 & 0.0000 \\
$\mathrm{C}(6)$ & 1.1341 & 0.0017 & 0.0000 \\
$\mathrm{Cl}(2)$ & -2.6023 & 0.9227 & 0.0000 \\
$\mathrm{H}(3)$ & -2.1658 & -1.8981 & 0.0000 \\
$\mathrm{H}(4)$ & 0.0000 & -3.1833 & 0.0000 \\
$\mathrm{H}(5)$ & 2.1658 & -1.8981 & 0.0000 \\
$\mathrm{Cl}(6)$ & 2.6023 & 0.9227 & 0.0000 \\
\hline
\end{tabular}

Energy $=-1165.73255$ Hartrees (corrected for ZPE). 
Figure S1 Experimental and difference (experimental minus theoretical) molecularscattering intensities for 1,2,3-trifluorobenzene, $\mathbf{1}$.

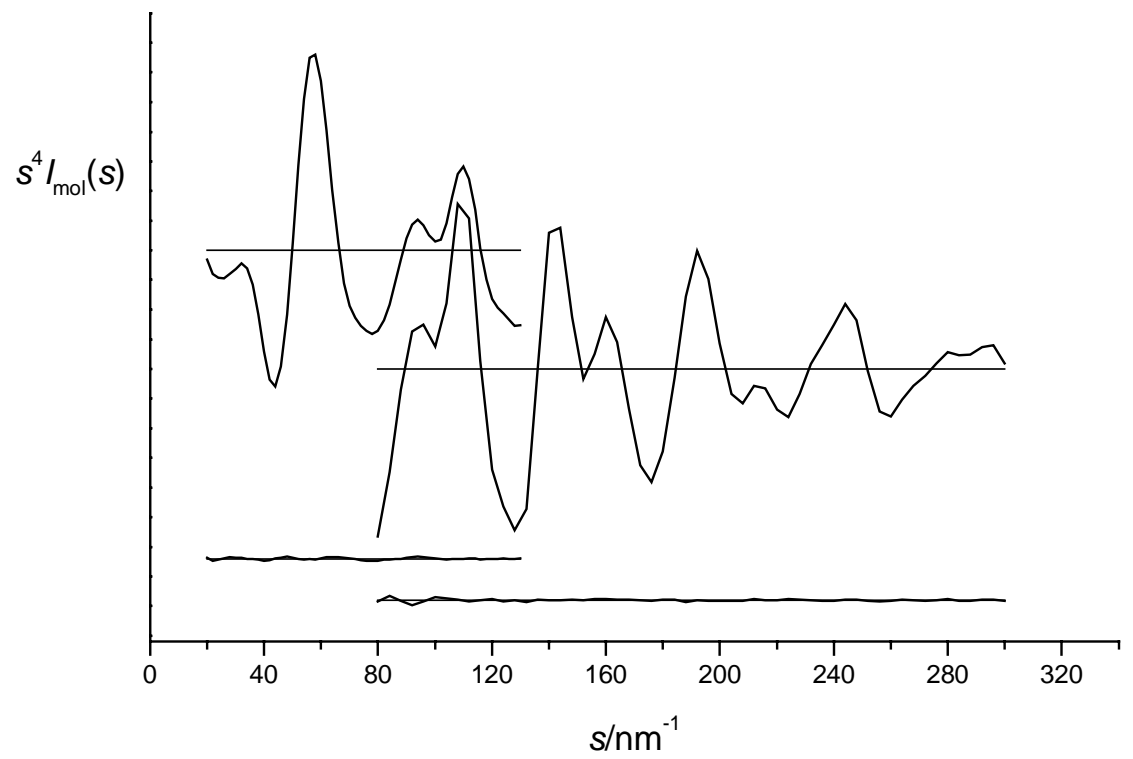

Figure S2 Experimental and difference (experimental minus theoretical) molecularscattering intensities for 1,3,5-trifluorobenzene, 2 .

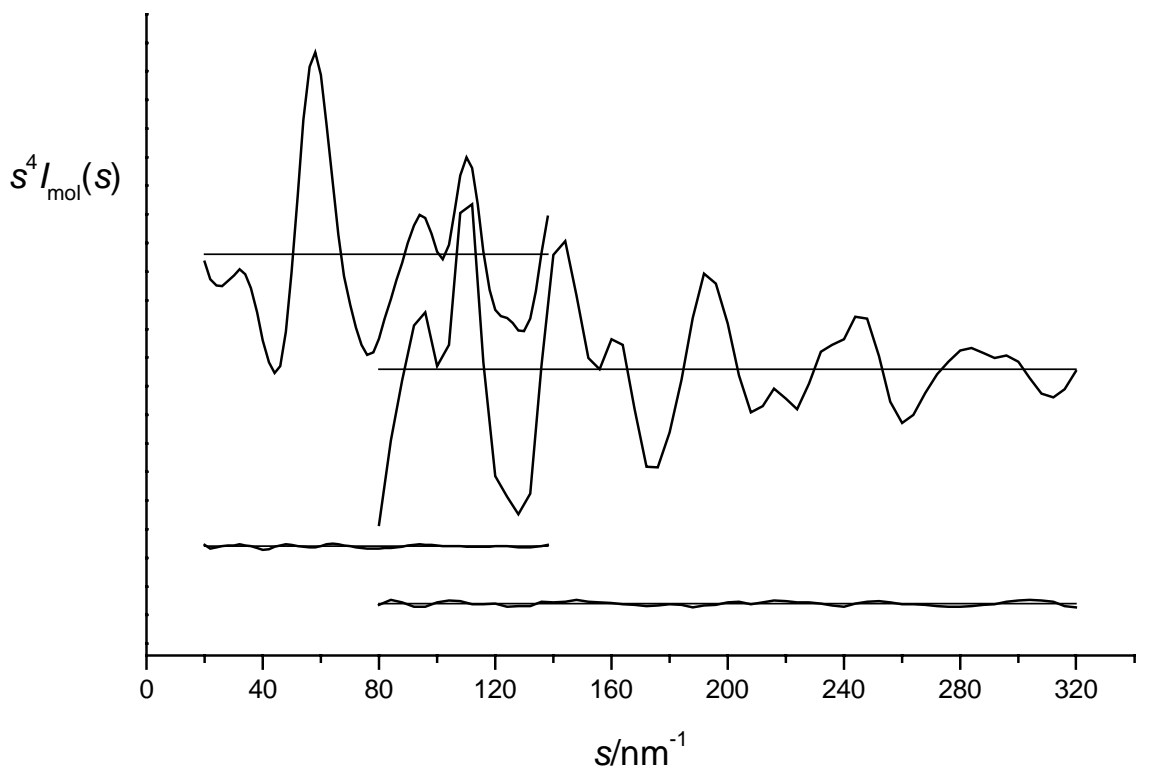


Figure S3 Experimental and difference (experimental minus theoretical) molecularscattering intensities for 2,6-difluoropyridine, 3.

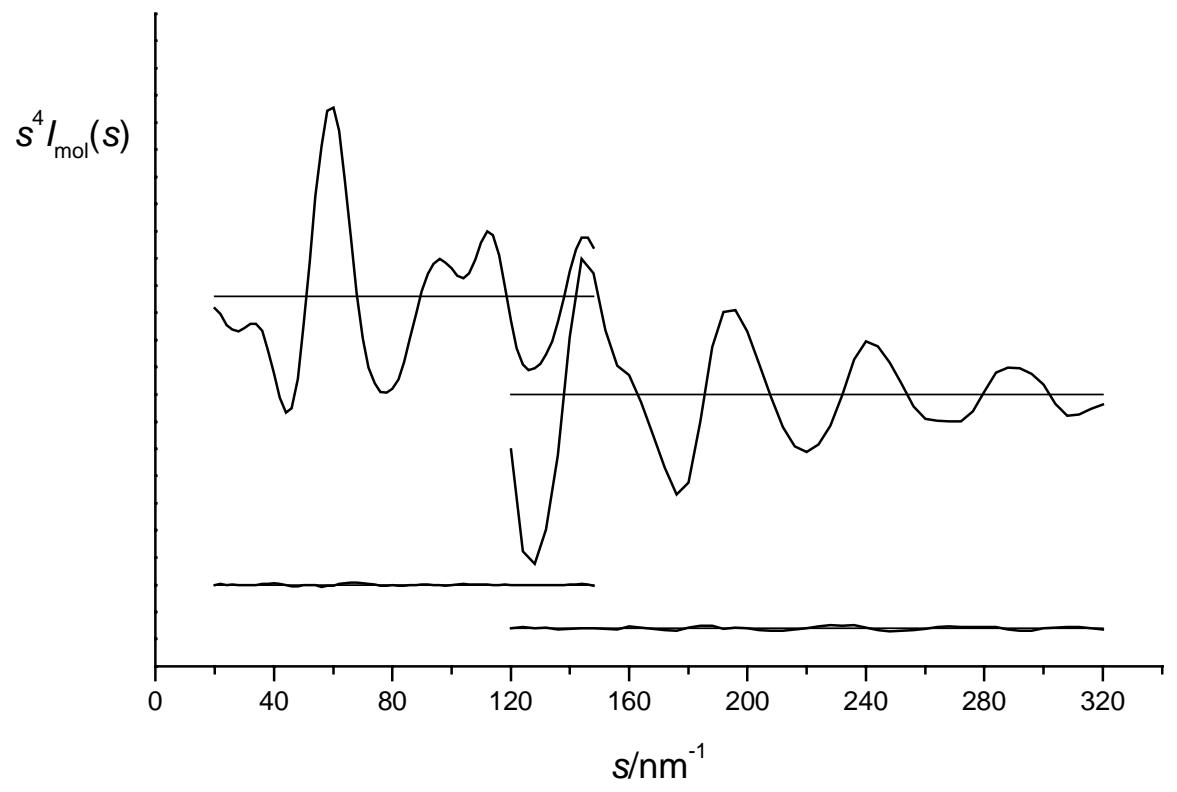

Figure S4 Experimental and difference (experimental minus theoretical) molecularscattering intensities for 2,6-dichloropyridine, 4.

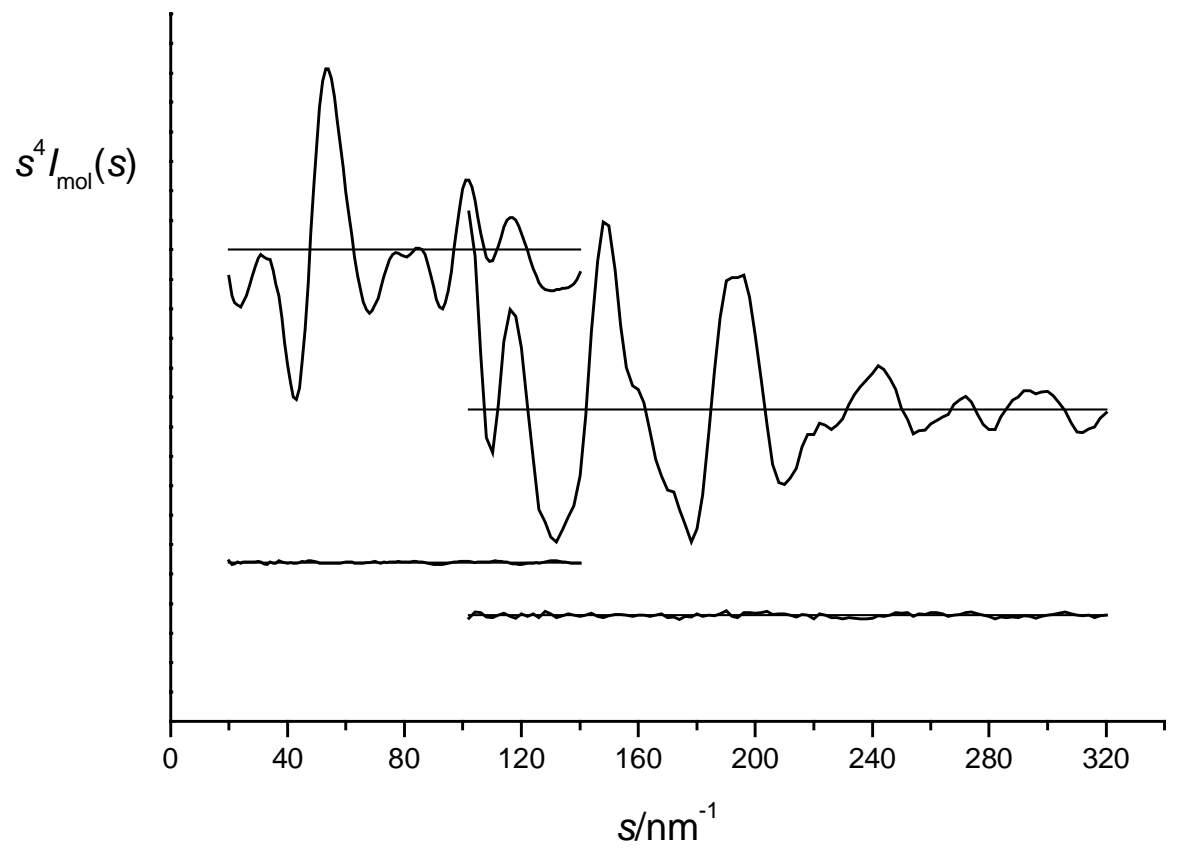




\section{Comparison of the results of this refinement of 1,3,5-trifluorobenzene with those from two previous refinements}

Of the three previous attempts to determine the GED structure of $\mathbf{2}$, the original one will not be considered, as it entirely ignored the deformation of the benzene ring. Parameters from the two most recent GED refinements and from this present study are compared in Table 4. It should be noted that each study reports a fundamentally different type of distance; to help with comparison, the $r_{\mathrm{a}}$ values for the bond lengths from the current study have been converted to $r_{\mathrm{g}}$ values $\left(r_{\mathrm{g}} \approx r_{\mathrm{a}}+u^{2} / r_{\mathrm{a}}\right)$, where $u$ is the root-mean-squared amplitude of vibration. Through using more accurate (calculated) amplitudes of vibration and by taking into account the effects of shrinkage by applying curvilinear corrections (giving $r_{\mathrm{h} 1}$ values) we have been able to obtain a value for $r \mathrm{C}-\mathrm{C}$ that compares favourably with the theoretical value. $\angle \mathrm{C}-\mathrm{C}(\mathrm{F})-\mathrm{C}$ is determined to within $0.3^{\circ}$ of the calculated value. Although the $\mathrm{C}-\mathrm{C}$ distance is close to the MP2 calculated value, the $\mathrm{C}-$ $\mathrm{F}$ distance determined in this work is more different than was the case in the previous study reported in reference 7 , although it lies within 2 standard deviations of that obtained from the study detailed in reference 6 . It is possible that the calculated value has not yet reached convergence and that at a higher level the value for $r \mathrm{C}-\mathrm{F}$ could be smaller.

\section{Comparison of the structures of 1 and 2 with those of their chloro analogues}

The GED structures of 1,2,3-trichlorobenzene ${ }^{23}$ and 1,3,5-trichlorobenzene ${ }^{24}$ have previously been published. Compared to 1, 1,2,3-trichlorobenzene shows very similar trends in internal ring angles and bond lengths. The only significant difference is in the $\mathrm{C}(2)-\mathrm{C}(1)-\mathrm{X}$ angle, which in the case of $1,2,3$-trichlorobenzene is very close to $120^{\circ}$ $\left[120.59(23)^{\circ}\right]$, whereas in $\mathbf{1}$ it is $118.4(3)^{\circ}$. The wider angle in the chlorine compound reflects steric repulsion between chlorine atoms on $\mathrm{C}(1)$ and $\mathrm{C}(2)$, which is manifested whenever there are chlorine substituents on adjacent atoms of benzene rings. For the 1,3,5-trisubstituted benzenes the $\mathrm{C}-\mathrm{C}$ bond lengths are identical in both the fluoro- and chloro-derivatives. The internal ring angles show that in $\mathbf{2}$ the angles are further from 
$120^{\circ}$ than they are in the 1,3,5-trichlorobenzene reflecting the greater electronegativity of fluorine. Effects of multiple fluorine substitution are more likely to be additive than those for chlorine substitution, because steric effects from adjacent substituents are much less significant.

\section{Comparison of the structures of 1 and 2 with those of other fluorobenzenes}

GED studies have previously been performed for other fluorinated benzenes, with trends similar to those in this paper reported. GED refinement of a $C_{2 \mathrm{v}}$-symmetric model of fluorobenzene ${ }^{25}$ gave ring angles that were in excellent agreement with those obtained from microwave spectroscopy, X-ray crystallography and liquid-crystal NMR spectroscopy. Notably, the results were not reproduced by ab initio MO calculations (Pulay's gradient method), ${ }^{26}$ which underestimated the angular distortion of the ring at the ipso carbon. The experimental angle (GED) at the ipso angle was $123.4(2)^{\circ}$, while the $a b$ initio calculations predicted $122.3^{\circ}$. We have now performed a higher-level MO calculation (MP2/6-311++G**), which gives the angle as $122.5^{\circ}$, still significantly narrower than the well-defined GED-determined angle.

In the GED study of 1,2,4,5-tetrafluorobenzene,${ }^{27}$ the benzene ring deviated only slightly from $D_{6 \mathrm{~h}}$ symmetry. The $\mathrm{C}-\mathrm{C}(\mathrm{F})-\mathrm{C}$ angle was $120.6(3)^{\circ}$, which is in agreement with the value of $120.4^{\circ}$ from an SCF ab initio calculation (and also in good agreement with an MP2/6-311++G** calculation performed by us, which gave $120.5^{\circ}$ ), but low when compared to values of $123.4(5)^{\circ}$ for fluorobenzene and $122.9(6)^{\circ}$ for $1,3,5$ trifluorobenzene (this study). The value is, however, close to those (120.2 and $\left.120.8^{\circ}\right)$ obtained from a calculation (MP2/6-311++ $\mathrm{G}^{* *}$ ) on 1,2,4-trifluorobenzene performed during this work. The reasons that we have already suggested for the narrow angles in 1,2,4-trifluorobenzene are reinforced by this result.

The GED study of hexafluorobenzene ${ }^{28}$ found that the $\mathrm{C}-\mathrm{C}$ bond length [139.4(7) pm] was, to within the error limits, the same as in benzene. As hexafluorobenzene has a fluorine atom at every substituent position, the effects of the fluorine atoms on lengthening $\mathrm{C}-\mathrm{C}$ where a fluorine is bound to one or both of the carbon atoms and on shortening $\mathrm{C}-\mathrm{C}$ bonds where no fluorine is bound directly to a carbon have cancelled out. 